Gerhard Leibholz (Herausg.) bzw. Peter Häberle (Herausg.)

Jahrbuch des öffentlichen Rechts, Neue Folge, Bände 29, 30, 31 und 32

J.C.B. Mohr (Paul Siebeck) Verlag, Tübingen, 1980, 718 S., DM 286,--; 1981, 707 S., DM 295,—; 1982, 387 S., DM 238,—; 1983, 674 S., DM 285,-

Mit der Edition von 30 Bänden des Jahrbuchs des öffentlichen Rechts hat sich Gerhard Leibholz große und bleibende Verdienste um die Rechtsvergleichung im öffentlichen Recht erworben; er hat eine Publikation geprägt, die ihresgleichen im deutschsprachigen Raum nicht hat. Nach Leibholz' Tod am 19. Februar 1982 ist der 31. Band noch nach seinen Dispositionen gestaltet worden. Neuer Herausgeber ist Peter Häberle, der den 1983 erschienenen Band 32 veranstaltet hat.

In dieser Zeitschrift sind viele Bände des Jahrbuches gewürdigt worden, ${ }^{1}$ sieht sie doch ihre Aufgabe in der Betreuung eines Ausschnitts des dort erfaßten thematischen Spektrums. Mit der Verfassungsentwicklung in den Staaten Afrikas, Asiens und Lateinamerikas befaßt sich das Jahrbuch in seiner von Band zu Band unterschiedlich umfangreichen Abteilung 'Entwicklung des Verfassungsrechts im außereuropäischen Bereich'. Vor allem diesem Bereich seien daher die folgenden Bemerkungen zu den vier zuletzt erschienenen Bänden gewidmet. Dabei handelt es sich in vier Bänden um 13 von insgesamt 61 Abhandlungen. Die Mehrzahl der Beiträge des Jahrbuches befaßt sich mit Themen aus dem europäischen Ausland, mit Untersuchungen zum Verfassungsrecht der Vereinigten Staaten und allgemeinen staatstheoretischen Problemen. Hinzu kommen vor allem Arbeiten zum Verfassungsrecht der deutschen Bundesländer und historisch orientierte Beiträge. Dabei scheint in Band 32 eine Absicht des neuen Herausgebers zu Tage zu treten, nach und nach ein möglichst vollständiges Mosaik zusammenzufügen: Das Verfassungsrecht keines der in jenem Band behandelten europäischen Staaten (Großbritannien, Finnland, Niederlande, Griechenland und Portugal) war in den sechs vorangegangenen Jahrbüchern systematisch vorgestellt worden, bei Portugal liegt dies sogar 24 Jahre zurück. Begrüßenswert ist auch, daß die Verfassungslage Griechenlands, dessen politische Geschichte nach der demokratischen Wiedergeburt geprägt ist durch ein unübersichtliches innenpolitisches Kräftespiel, das zudem den deutschen Rechtsvergleicher nicht nur wegen des durch die europäische Integration intensivierten Kontakts, sondern auch wegen der Tradition enger persönlicher Kooperation zwischen griechischen und deutschen Verfassungsrechtlern in besonderem Maße interessiert, in gleich drei Abhandlungen analysiert wird, die zusammen ein äußerst informatives Gesamtbild geben: Kaltsojia-Tournavitis historische Einführung; Dagtoglous Präsentation der Verfassung von 1975 und Frau Iliopoulos-Strangas' dichte Studie über den Grundrechtsschutz in Griechenland. Derartiges editorisches Zusammenordnen sich ergänzender

l Band 20 (1971), VRU 6(1973), S. 251 (von Wedel); Band 21 (1972), VRU 6(1973), S. 500 (Krakau); Band 22 (1973), VRU 7 (1974), S. 357 (von Wedel); Bände 23 bis 26 (1974 bis 1977), VRU 12 (1979), S. 165 (Kunig); Band 27 (1978), VRU 13 (1980), S. 161 (Kunig); Band 28 (1979), VRU 13 (1980), S. 398 (Kunig). 
Beiträge ist vorbildlich und sollte bei allen bekannten Schwierigkeiten weiterhin erstrebt werden.

Der Kontinent Lateinamerika ist in den hier vorzustellenden vier Jahrbüchern durch Beiträge unterschiedlichen Zuschnitts vertreten. Der Argentinier Pablo A. Ramella gibt einen Bericht über die /Verfassungsentwicklungı seines Landes - von 1968 bis 1979 (Band 29) - ein Aufsatztypus, wie er lange Zeit für das Jahrbuch charakteristisch war. Eine kürzere Darstellung desselben Autors widmet sich dem Problem des Föderalismus in Argentinien (Band 31). Dieter Blumenwitz' Darstellung der chilenischen Verfassung von 1975 (Band 30, mit Text in deutscher Úbersetzung) steht für einen weiteren Typ von Jahrbuch-Beiträgen: die vorwiegend informierende Vorstellung einer neuen Verfassung, samt historischer Einleitung und rechtspolitischer Bewertung. Letztere fällt bei Blumenwitz in diesem Falle vorsichtig-werbend aus. Hans-Rudolf Horn, neben Hector Fix-Zamudio und Friedrich Wehner wohl derjenige, der im deutschsprachigen Raum am meisten zur Kenntnis des mexikanischen Verfassungsrechts beigetragen hat, ${ }^{2}$ gibt in Band 29 eine Skizze von dessen 'Grundzügen', wobei er die sowohl in der innermexikanischen Diskussion wie auch für die vergleichende Betrachtung als die interessantesten Gegenstände angesehenen Phänomene in den Vordergrund stellt: die Frage sozialer Grundrechte, den Verfassungsgerichtsschutz des Bürgers (Amparo) und die mexikanische Spielart des Präsidialismus. Derartige Schwerpunktbildung anhand des politischen und Vergleichungs-Interesses ist gewiß einer Orientierung rein an Verfassungstexten vorzuziehen, wie sie insbesondere in früherer Zeit häufig im Jahrbuch zu beobachten war.

Waldemar Hummer schließlich berichtet - gleichfalls in Band 29 - über den fortschreitenden Integrationsprozeß in Lateinamerika.

Zweimal Indien, zweimal Japan, Burma und Papua-Neuguinea sind die behandelten asiatischen Staaten; zu nennen ist hier auch Ernst E. Hirschs Einführung in die neue türkische Verfassung von 1982 (Band 32), die deren Text und denjenigen des Parteiengesetzes von 1983 dokumentiert. Die auf Indien bezogenen Beiträge stammen von dem früheren Supreme Court-Richter J.L. Kapur, der schon wertvolle Darstellungen über den Supreme Court of India (Bände 11, 24), die Stellung des Staatspräsidenten (Bände 14, 25), die indische Verfassung insgesamt (Band 23) und ihre föderalen Aspekte (Band 26) für das Jahrbuch geliefert hat. Diesmal ergänzt er den erwähnten Föderalismus-Beitrag vor dem Hintergrund der Rückkehr Indira Gandhis an die Spitze der

2 Vgl. H. Fix-Zamudio, Verfassungskontrolle in Lateinamerika, JöR 25 (1976), S. 649; ders. México: El Organismo Judicial, VRU 10 (1977), S. 391; F. Wehner, Der mexikanische Präsidialismus und die Verfassung, VRU 10 (1977), S. 381; ders., Grundlagen einer mexikanischen Verfassungsgeschichte, Beiheft zu VRU, 1978 (rezensiert von Scheffler, VRU 13, 1980, S. 89); H.-R. Horn, Das Amparo-Verfahren in Mexiko, VRU 1 (1968), S. 162; ders., Staatsrechtsdenken und Verfassungsvergleichung in Mexiko, VRU 10 (1977), S. 461; s. ferner die Rezensionen von Horns rechtsvergleichender Mexiko-Studie Legitimation und Grenzen der Exekutive (VRU 13, 1980, S. 178 - Scheffler) bzw. von Fix-Zamudios Schrift über Verfassungsgerichtsbarkeit und Menschenrechtsschutz (VRU 14, 1981, S. 216 - Horn) und schließlich die einschlägigen Tagungsberichte von Fix-Zamudio, VRU 10 (1977), S. 495, und Horn, VRU 14 (1981), S. 101. 
Zentralregierung und erörtert das prekäre Verhältnis von Zentralregierung und Länderregierungen bzw. -parlamenten, dessen Relevanz für Indien im Jahr 1984 eher noch gewachsen ist (Band 29). In Band 31 widmet sich Kapur erneut der Stellung des Staatspräsidenten, diesmal im Verhältnis zum Council of Ministers.

Kenji Hirota skizziert in Band 29 das Gerichtssystem in Japan, wobei leider die verfassungsrechtliche Stellung der Richter nur angedeutet wird. Was richterliche Unabhängigkeit` in Japan heute bedeutet, wie 'Verfahrensöffentlichkeit` abläuft, würde man gern näher erfahren und mit den japanischen Justiztraditionen konfrontiert sehen. Ergiebiger ist Nozumu Shimizus Studie zum 'Tenno-System', die auch auf die jüngst viel diskutierte Frage des Verhältnisses von Staat und Religion in Japan recht ausführlich eingeht. ${ }^{3}$

Klaus Fleischmann gibt in Band 31 einen Uberblick über den Regelungsinhalt der Verfassung Burmas von 1974, zu der er 1976 eine Monographie vorgelegt hat. ${ }^{4}$ Gerade bei Staaten, über deren reale politische Machtstrukturen hierzulande so wenig bekannt ist wie im Falle Burmas hinterläßt die Lektüre eines eher 'verfassungstextlich' konzipierten Beitrags ein zwiespältiges Gefühl. Auf die Verfassungswirklichkeit durchzustoßen, ja auch Akteure, deren Bestrebungen und Handlungen, anzuführen, wie es etwa für einige der Indien-Beiträge Kapurs charakteristisch ist, wäre gerade hier gewiß hilfreich.

Ebenfalls vorwiegend referierend ist Wilhelm Wenglers Beitrag über die Verfassung Papua-Neuguineas angelegt, an der Wengler insbesondere die den obersten Gerichten eingeräumte Rechtsfortbildungskompetenz interessiert. Die ungewöhnlich detaillierte Verfassung des pazifischen Staates, Musterbeispiel des Problems Verfassungsgebung für unabhängig werdende Staaten ohne autochthone Verfassungstradition, hatte schon bald nach ihrer Entstehung erhebliches rechtsvergleichendes Interesse ausgelöst - eine Diskussion, an die Wengler allerdings keinen Anschluß sucht - insgesamt durch zögernde Formulierungen eher den Eindruck erweckend, als sei sein Thema fremdartig und kaum bearbeitet. $^{5}$

Es bleibt der Kontinent Afrika. Leider nur ein Beitrag ist hier anzuzeigen, derjenige van Wyks über richterliche Kontrolle in Südafrika (Band 29), der den Fragenkreis außerordentlich detailliert abschreitet und eine präzise Beschreibung gibt. Rubins bzw. Schollers Beiträge über Ghana bzw. Äthiopien, die 1976 im Jahrbuch erschienen sind, haben somit bisher noch keine Fortsetzung gefunden, was af rikanische Themata angeht, die nicht der Republik Südafrika gewidmet sind (dazu noch Zimmermann in Band 27).

3 Vgl. R. Heuser, Der Grundsatz der Trennung von Staat und Religion in Japan, VRU 16 (1983), S. 67.

4 K. Fleischmann, Die Neue Verfassung der Union von Birma. Vorgeschichte, Inhalt, Wirkung, 1976.

5 Was nicht der Fall ist, vgl. nur A. Dix, Papua-Neuguinea, in: Bryde/Hecker/Hernekamp, Verfassungstexte, Beilage zu VRU 3/1976; J. Golding, The Constitution of Papua New Guinea, 1978, rezensiert von Dix in VRU 12 (1979), S. 429; J. Golding, Legalism Rampant: The Heritage of Imposed Law and the Constitution of Papua New Guinea, VRU 12 (1979), S. 223; B. B. Sakora, Judicial Law-Making and the Papua New Guinea Constitution, in: P. Sack (ed.), Pacific Constitutions, 1980, S. 257; neuerdings noch Sack, "Decolonisation", "Independence Constitutions" and the "Modern State" in the Pacific Islands, VRU 17 (1984), S. 5. 
Diese Vernachlässigung afrikanischer Staaten, deren ıobjektive` Ursachen bekannt sind, erkennt man als allgemeines Forschungsproblem auch anhand der sehr nützlichen Bibliographie deutscher Dissertationen zum ausländischen öf fentlichen Recht, die Peter M. Heer für Band 31 zusammengestellt hat. Zwischen 1972 und 1980 verzeichnet sie insoweit eine einzige, nämlich dem Kirchenrecht des frankophonen Schwarzafrikas gewidmete Arbeit!

Es sei gestattet, aus der Sicht dieser Zeitschrift eine Erwartungshaltung an die künftige Gestalt der aus der Verfassungsvergleichung nicht wegzudenkenden Institution 'Jahrbuch des öf fentlichen Rechts` zu formulieren: Danach wäre wünschenswert mehr Raum für außereuropäische Entwicklungen, mehr Raum insbesondere für afrikanisches Verfassungsrecht (man denke nur an Nigeria, den Sudan, Senegal, Tansania, Marokko), mehr Raum für Beiträge, die die Analyse von Verfassungstexten transzendieren, mehr systematische Beiträge als Úberblicksartikel, mehr Raum für Beiträge, die die rechtswissenschaftliche Entwicklungsländerforschung (die etwa in den Vereinigten Staaten und in Frankreich ein quantitativ und qualitativ weit höheres Niveau gewonnen hat als hierzulande) zur Kenntnis nimmt und auf sie reagiert: Die deutsche Verfassungsrechtsvergleichung kann auf einen derartigen Beitrag des Jahrbuchs für öffentliches Recht nicht verzichten.

Und schließlich: Es wäre zu begrüßen, wenn Peter Häberle, der sich wie kaum ein zweiter in Praxis und Theorie um das Rezensionswesen verdient gemacht hat, auch in das Jahrbuch einen Rezensionsteil aufnähme. Die Fülle einschlägiger Veröffentlichungen kann von den wenigen parallel interessierten Zeitschriften nicht bewältigt werden.

Philip Kunig

\section{Uwe Wesel}

\section{Juristische Weltkunde. Eine Einführung in das Recht}

Suhrkamp Taschenbuch Wissenschaft Nr. 467,

Suhrkamp Verlag, Frankfurt am Main, 1984, 213 S. DM 14,-

Der Titel des Buches bedarf der Erläuterung. Wesel gibt nicht eine Einführung in die Rechtsvergleichung, sondern schildert die "Welt" der Juristen, das, was sie seiner Ansicht nach denken und das, was sie tun. Recht definiert er als "das, was in den Köpfen von Juristen vor sich geht" (S. 7). Dem Text liegt eine Vorlesung für Erstsemester zugrunde.

$\mathrm{Da}$ Wesel auch rechtshistorische und rechtsanthropologische Interessen hat, findet an vielen Stellen seinen Niederschlag. Evans-Pritchards Nuer-Studien werden vorgeführt, um einen Kontrapunkt zu den Strukturen der Rechtsordnung im modernen Industriestaat zu finden. Antikes, mittelalterliches, frühkapitalistisches, nationalsozialisti- 